\title{
Téoros
}

Revue de recherche en tourisme

\section{Politiques de communication et identifications territoriales différenciées}

\section{Les usages politiques des vagues et de l'univers du surf par les municipalités de la côte Aquitaine}

\section{Christophe Guibert}

Volume 25, numéro 2, été 2006

URI : https://id.erudit.org/iderudit/1071072ar

DOI : https://doi.org/10.7202/1071072ar

Aller au sommaire du numéro

Éditeur(s)

Université du Québec à Montréal

ISSN

0712-8657 (imprimé)

1923-2705 (numérique)

Découvrir la revue

Citer cet article

Guibert, C. (2006). Politiques de communication et identifications territoriales différenciées : les usages politiques des vagues et de l'univers du surf par les municipalités de la côte Aquitaine. Téoros, 25(2), 62-71.

https://doi.org/10.7202/1071072ar d'utilisation que vous pouvez consulter en ligne. 


\title{
Politiques de communication et
} identifications territoriales différenciées

\author{
Les usages politiques des vagues et de l'univers du surf \\ par les municipalités de la côte Aquitaine
}

\section{Christophe Guibert}

Le sport est souvent mobilisé par les élus locaux français lors de la construction d'une identification territoriale ${ }^{1}$. Plus particulièrement, la vague et l'univers du surf sur la côte Aquitaine, sport qui constitue le support de l'analyse, formalisent un « imaginaire territorial » et accentuent le dynamisme politique qui y est lié. Le surf « contribue à alimenter une certaine image: on vient à Lacanau parce que la mer est belle, parce que les surfeurs, parce que la festivité... ça devient attractif »2, précise Gibus de Soultrait, rédacteur de Surf Session, principale revue spécialisée de surf en France. Si le surf est « attractif », c'est que les représentations collectives se construisent à travers des éléments discursifs et des identifications marquantes. L'article du quotidien national Le Monde daté de décembre $2000^{3}$, composé de réponses de Jean-Pierre Augustin, insiste par exemple sur le rapport étroit entretenu par l'Aquitaine et l'ensemble des collectivités locales du littoral avec le surf, ce qui, selon l'auteur, renforce «les dynamiques territoriales ». D'après ce géographe, les collectivités locales dans leur ensemble « ont bien compris » l'intérêt du surf, activité qu'elles semblent soutenir.

Le surf est un sport très structurant de l'offre touristique sur le littoral aquitain, de l'échelle communale à la région. C'est en effet en Aquitaine, dans les départements de la Gironde, des Landes et des PyrénéesAtlantiques, que la pratique du surf est la plus développée en Europe. Les catégories d'acteurs avec lesquelles les élus locaux ont affaire dans la définition de «territoires touristiques » (Corneloup et al., 2000) sont dès lors nombreuses. Le siège de la Fédération française de surf (FFS), un maillage associatif dense (avec près de $80 \%$ des licences nationales), la majorité des structures com- merciales (écoles, magasins spécialisés, ateliers de fabricants de planches, shapers), ainsi que les sièges des entreprises de surfwear contribuent à qualifier la côte aquitaine - selon la presse économique et la Chambre de commerce et d'industrie de BayonnePays basque - de "Surficon Valley ", à l'image de la Silicon Valley en Californie.

Après avoir défini les caractéristiques des vagues de la côte Aquitaine, en tant que ressources naturelles et support de la pratique du surf, nous verrons que les productions d'externalité et les stratégies de développement touristiques par les municipalités à l'aide de ces matérialités naturelles sont différenciées. Une politique municipale "surfique » est produite à partir des propriétés communales et de la dimension temporelle de l'existant, c'est-à-dire de l'historicité des ressources disponibles localement (Guibert, 2004 : 217). En conséquence, la révélation et la valorisation de la vague et du surf, en tant que substrat idéologique du territoire communal sur la côte Aquitaine, ne sont homogènes ni sur le plan politique ni sur le plan de l'identification territoriale. Toutefois, la finalité de ces actions politiques est sensiblement la même, à savoir: asseoir une identification suffisamment puissante pour alimenter l'offre touristique en direction des vacanciers potentiels.

\section{Méthode et cadre conceptuel}

Pour justifier les résultats apportés dans ce travail, des entretiens ont été menés de 2000 à 2005 auprès d'élus locaux (maires, adjoints chargés des affaires sportives ou de la communication, conseillers municipaux) et de responsables de clubs de surf. L'usage des matériaux qualitatifs a permis d'avancer des explications en accordant une place importante aux dispositions et aux éléments discursifs sur les pratiques des agents sociaux.

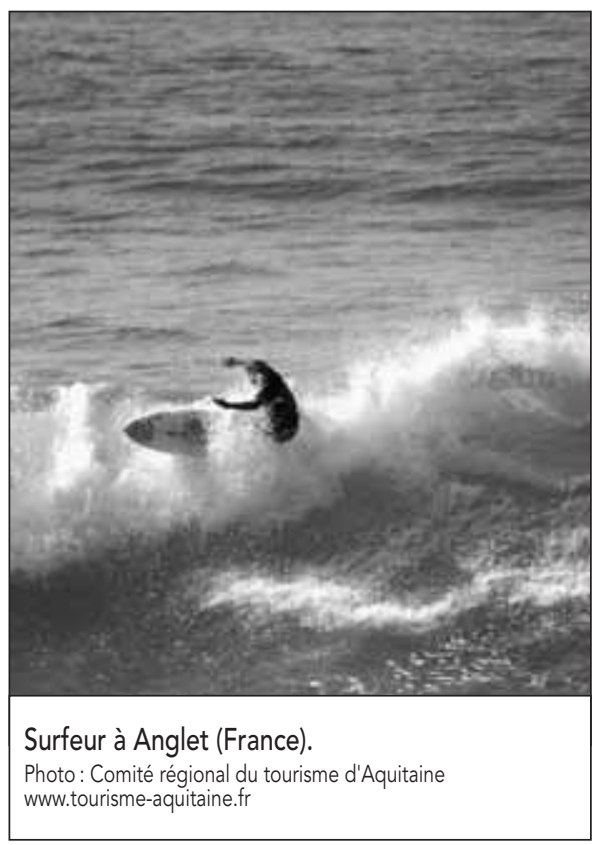

Les ressources documentaires se révèlent également être d'une grande richesse. Les événements locaux, par exemple, sont largement retranscrits sur Internet et dans les «pages locales» de la presse régionale, comme le quotidien Sud-Ouest ; ailleurs, les contenus et les couvertures (photographies, titres) des magazines municipaux servent de vitrines aux collectivités locales dont ils sont issus ; enfin, les livres de surf et la presse spécialisée (Surf Session, Surf-Time) représentent une mine d'information difficilement "occultable", ne serait-ce que pour mieux les déconstruire et en saisir les effets sur les politiques municipales. L'étude de ces documents est un bon moyen d'appréhender les logiques d'actions politiques dans la définition d'une identification territoriale.

L'ambition d'analyser les singularités des politiques municipales des communes du littoral aquitain en matière de surf requiert le 
choix d'un terrain d'étude relativement étendu pour qu'il présente des caractéristiques historiques et socioéconomiques variées. Concernant trois départements de la façade atlantique (Gironde, Landes et Pyrénées-Atlantiques), l'espace ou le " champ politique » (Bourdieu : 2000) privilégié de l'enquête se situe entre l'embouchure de la Gironde et la frontière espagnole, soit de Lacanau à Hendaye. II englobe des espaces balnéaires "touristifiés " de façon plus ou moins tardive. Le recours à l'étude de cas s'est révélé une solution adaptée pour décrypter les mécanismes politiques et les enjeux liés au développement du surf. Les résultats obtenus, non exhaustifs mais néanmoins significatifs des possibilités d'actions politiques, s'observent pour des communes à des moments précis, ceux de l'investigation. Les références multidisciplinaires en géographie, en tourisme, en économie, en sociologie et en sciences politiques permettent de saisir les stratégies politiques de construction symbolique du territoire communal.

D'un point de vue conceptuel, l'idée de " développement territorial » (Gumuchian et Marois, 2000) s'impose comme une figure nouvelle du développement et de l'aménagement des économies locales. Dans une logique de valorisation touristique, les stratégies des élus politiques locaux participent à

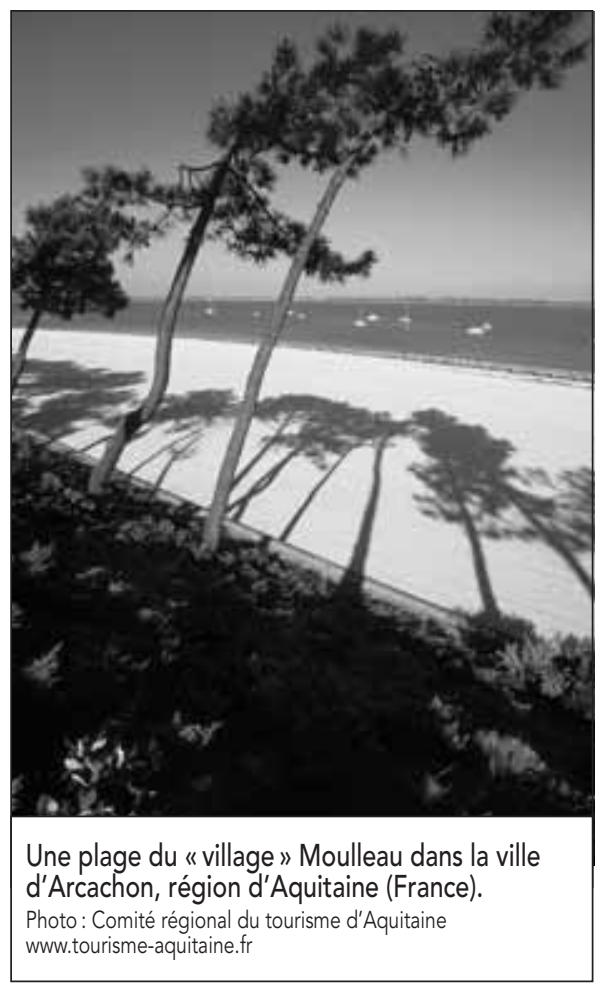

la construction du territoire. Pour autant, le processus de territorialisation ne peut être exclusivement réduit aux stratégies politiques. En effet, les élus, dans leurs actions, manipulent inévitablement la notion de "ressource territoriale » (Gumuchian et Pecqueur, 2004 : 4). Élément caractéristique et construit d'un territoire spécifique dans une optique de développement et de production d'externalité, la ressource territoriale renvoie à une intentionnalité politique, en même temps qu'au substrat idéologique du territoire. Elle se définit à partir de composantes matérielles, par exemple le patrimoine ou le paysage, et à partir de composantes idéelles, entre autres l'authenticité et la profondeur historique. "Produire du territoire », c'est donc élaborer des projets adaptés, aux yeux des élus locaux, à des réalités locales.

Le territoire se définit à partir des matérialités qui le structurent, puis des propriétés symboliques qui font que ce dernier est chargé de sens pour ceux qui y vivent et ceux qui le traitent. D'une part, les productions du territoire par l'action politique sont donc influencées par les lieux dans lesquels elles se conçoivent (l'histoire du surf, le maillage associatif, les vagues, les lieux de pratique reconnus, etc.) et, d'autre part, elles marquent les lieux dans lesquels elles se déroulent. Les "particularismes locaux" (Callède, 2002 : 206) sont en ce sens au centre des préoccupations des équipes municipales. L'enjeu politique, plus que le soutien au surf, est le développement de la commune qui correspond le plus souvent à une logique de «marketing territorial » (Le Bart, 1999 : 415). II en résulte la production de ville de surf ou de "surfurbia » (Augustin, 1997 : 125), c'est-à-dire une commune où le surf est intégré aux institutions locales, tout en influençant l'aménagement, l'urbanisme et l'identification territoriale.

Avant d'aborder les choix des élus locaux relatifs aux orientations politiques menées, il convient de préciser les caractéristiques de l'univers du surf en France, à savoir "l'immatérialité » de la pratique et « la malléabilité » des représentations de cet univers (Guibert : 2005). Ces caractéristiques sont à l'origine de la singularité de l'usage politique d'une activité comme le surf à l'égard des autres sports, puis de la diversité des actions menées par les municipalités sur la côte aquitaine.

\section{Produire du territoire avec de "l'immatériel »}

Les propriétés organisationnelles et spatio-temporelles du surf rompent avec celles du sport en général. En effet, la pratique du surf ne suppose aucune infrastructure puisque la vague, en tant que ressource naturelle, est le support de l'activité. Le lieu de pratique peut également changer en fonction des conditions climatiques. Les bancs de sable, par exemple (particulièrement dans le département des Landes), qui contribuent fortement à la qualité des vagues - évaluée comme telle dans la presse spécialisée -, changent d'emplacement au gré des tempêtes. Des vagues de qualité peuvent se situer sur les plages d'une commune et, à la suite d'une tempête ou de grands coefficients de marée, sur les plages de la commune voisine. Les conditions de pratique sont donc très dépendantes du climat, de la houle et du vent. Finalement, le surf est une activité qui se pratique dans des conditions éphémères et s'oppose en quelque sorte à des activités sportives qui se déroulent dans des conditions spatiotemporelles normalisées et récurrentes comme un stade, un circuit automobile, un parcours de golf, etc. La FFS estime qu'il y a près de 100000 adeptes en été sur les côtes du pays ; on comprend que les retombées touristiques de cette activité (cours dans les écoles privées de surf ${ }^{4}$, fréquentation en termes d'hébergement, de restauration etc.) ne peuvent a priori laisser les municipalités de la côte Aquitaine indifférentes. Le surf ne peut en outre susciter des émotions collectives au sens de Norbert Elias (1998), à l'instar du football, sport où les équipes représentent symboliquement beaucoup plus que de simples équipes (image de la ville, visibilité) ; tout au plus, il peut engendrer des représentations territoriales liées à la qualité des spots et aux lieux de manifestations suffisamment médiatiques. Le surf n'est pas en enjeu national qui permet de réactiver une sensibilité nationaliste propre aux manifestations sportives comme les Jeux olympiques et les championnats internationaux de sports collectifs (Coupe du monde de football, Coupe Davis en tennis, etc.), avec leurs symboles (le drapeau, l'hymne) et les discours qui leur sont liés (journalistiques et politiques notamment). L'absence de champion français à l'échelle internationale ou de compétitions interclubs reconnues rend difficile, à 
l'inverse de l'équipe de football ou de basket-ball locale, une identification territoriale légitime et puissante aux yeux des élus et de la population locale.

Marquer une ville avec une activité comme le surf exige donc des choix et des actions politiques singuliers. Premièrement, il s'agit, pour un élu du littoral, de construire symboliquement du territoire, c'est-à-dire une ressource "terrienne ", avec une ressource "aquatique " (la vague), ce qui, finalement, ne va pas systématiquement de soi. Deuxièmement, marquer un territoire durablement avec une activité éminemment estivale, dont les manifestations emblématiques (compétitions professionnelles, festivals) se déroulent en été, exige de la part des élus des actions différenciées, qui doivent en quelque sorte être sans cesse redéfinies. Prendre le surf comme objet dans la production symbolique du territoire engage donc un éclairage original sur le plan des usages politiques des ressources territoriales. Troisièmement, bien que faiblement pratiqué à l'échelle fédérale (moins de 20000 licenciés en 2003 selon la FFS), le surf suscite malgré tout un intérêt sensible chez les touristes en saison estivale. Les retombées touristiques engendrées sont importantes et contribuent par là même à une certaine forme de " développement local (Pecqueur, 2000). Les industries du surf telles que la fabrication et la vente de planches, mais aussi et surtout la consommation d'habits de surf, le surfwear, sont plébiscités par l'arrivée de milliers de surfeurs dans les stations côtières, qui se métamorphosent alors en "villes de surf ». Le surf, c'est également une économie florissante à en lire les magazines économiques et les courbes des actions de quelques entreprises. N'ayant en réalité que peu de rapports directs à proprement parler avec la pratique du surf, ces entreprises de surfwear (vêtements de surf), telles Quiksilver, Billabong ou Rip Curl, sont des multinationales australiennes et américaines dont les sièges européens se situent tous sans exception en Aquitaine. L'univers du surf s'apparente en conséquence à une multitude de représentations, ce qui lui confère en quelque sorte un caractère malléable. Aussi, les municipalités définissent-elles des politiques au regard de ce qui est, à leurs yeux, susceptible d'intéresser le développement de leur commune.

\section{La " malléabilité " de l'univers du surf et des vagues au profit des stratégies de marquage territorial}

La production de territoire est associée à une instrumentalisation de l'espace ${ }^{5}$ du surf, notamment à des fins de développements médiatique, économique, touristique, social et parfois sportif. Ce type d'action politique touche la mobilisation de la vague, du surf et plus généralement de l'univers du surf, c'est-à-dire la pratique, mais aussi et surtout les grands événements (festivals, compétitions professionnelles), les emplois directs (évalués à plus de 3000 pour la région), les représentations, etc. La production du territoire à l'aide de ce substrat singulier devient légitime aux yeux des élus, des médias et in fine des touristes et des investisseurs, lorsque l'identification communale qui en résulte est durable et originale. Cette originalité qui favorise une "différenciation des lieux » est possible, puisque l'univers du surf, qui ne correspond en rien à une unité nominale objective, est malléable. C'est cette malléabilité qui intéresse ici, car elle constitue une caractéristique qui, à l'inverse des activités sportives "traditionnelles", plus homogènes, est prise en compte par les élus locaux du littoral aquitain. Cette caractéristique rend possible des justifications et des choix qui sont divers, pour peu que l'analyse se fasse à l'échelle locale. L'univers du surf, comme toute pratique symbolique, est ce que les élus locaux qui tentent de le contrôler sur leur commune en font. En effet, une commune est un territoire construit par des propriétés intrinsèques que l'élu ne peut modifier. La taille, la population, les caractéristiques sociales, les références historiques sont autant de données avec lesquelles un élu doit combiner pour définir ses choix et ses orientations politiques.

Marquer une commune à l'aide du surf ou plus simplement proposer une action politique à l'égard du surf requiert une analyse fine des politiques municipales et des agents qui les définissent. Les premières lignes de la préface de l'ouvrage d'Alain Gardinier, Les Tontons Surfeurs, aux sources du surf français, proposées par un "écrivain du surf », Hugo Verlomme, résument une singularité du surf, sa malléabilité. Ce particularisme doit être pris en compte pour saisir les usages politiques liés à une telle activité :
Soyons honnêtes : personne n'aurait cru, dans la France des années 1950, que deux générations plus tard, le surf serait devenu un sport passion, une mode déclinée à l'infini, un style de vie, de vêtements, un Eldorado financier qui bouleverserait des régions entières, bénies par les vagues. Toujours à l'avant-garde, le surf est même devenu le mot de passe de la révolution Internet, le voici qui explose dans la publicité, envahit la mode, devient une manne financière, une pompe à sponsors, mais aussi un sport de haut niveau, une philosophie, un art de vivre, un sujet d'inspiration pour les cinéastes, les photographes, les peintres et dessinateurs, les musiciens et les écrivains, une passion montante pour les nouvelles générations [...] (Gardinier, 2004 : 7).

«Le » surf est une expression qui uniformise une réalité hétérogène et complexe. Les modalités de pratique - la compétition professionnelle ou amateur, le free surf professionnel ou amateur, etc. - sont très variées. La population de surfeurs en France répond à des variations en termes de profil sociologique, à savoir de génération, de genre ou encore d'origine sociale. Composite, elle ne peut être homogénéisée. Ainsi, des journalistes voient dans cette activité une pratique sportive amateur ou professionnelle, un moyen de s'évader par le voyage, un art plus qu'un sport ; des élus locaux y voient un simple jeu de plage, un outil touristique, des pratiquants socialement déviants ou un secteur vecteur de création d'emplois; des surfeurs y voient une activité écologique aux valeurs écologistes, un style de vie, un sport de compétition; etc. Ce principe de malléabilité, qui renvoie à de multiples significations, oriente avec force l'objet de ce travail, à savoir l'analyse des politiques municipales du littoral aquitain qui traitent d'une activité finalement singulière. Les cartes de l'Aquitaine proposées (fig. 1-4) permettent de mieux localiser ces variables qui sont souvent à l'origine d'un dynamisme politique particulier ou en sont la conséquence. Des indicateurs relatifs à l'univers du surf y sont représentés. On voit nettement une forte concentration d'activités dans le sud de l'Aquitaine, comme la localisation de la fédération, les sièges des entreprises de surfwear (fig. 3), une formation universitaire, des compétitions professionnelles et le siège européen de l'Association of Surfing Professionals (fig. 2), des ateliers de shapers (fig. 4), etc. dans le sud des Landes et dans les Pyrénées-Atlantiques, alors que 
Localisation d'indicateurs matérialisant l'univers du surf en Aquitaine
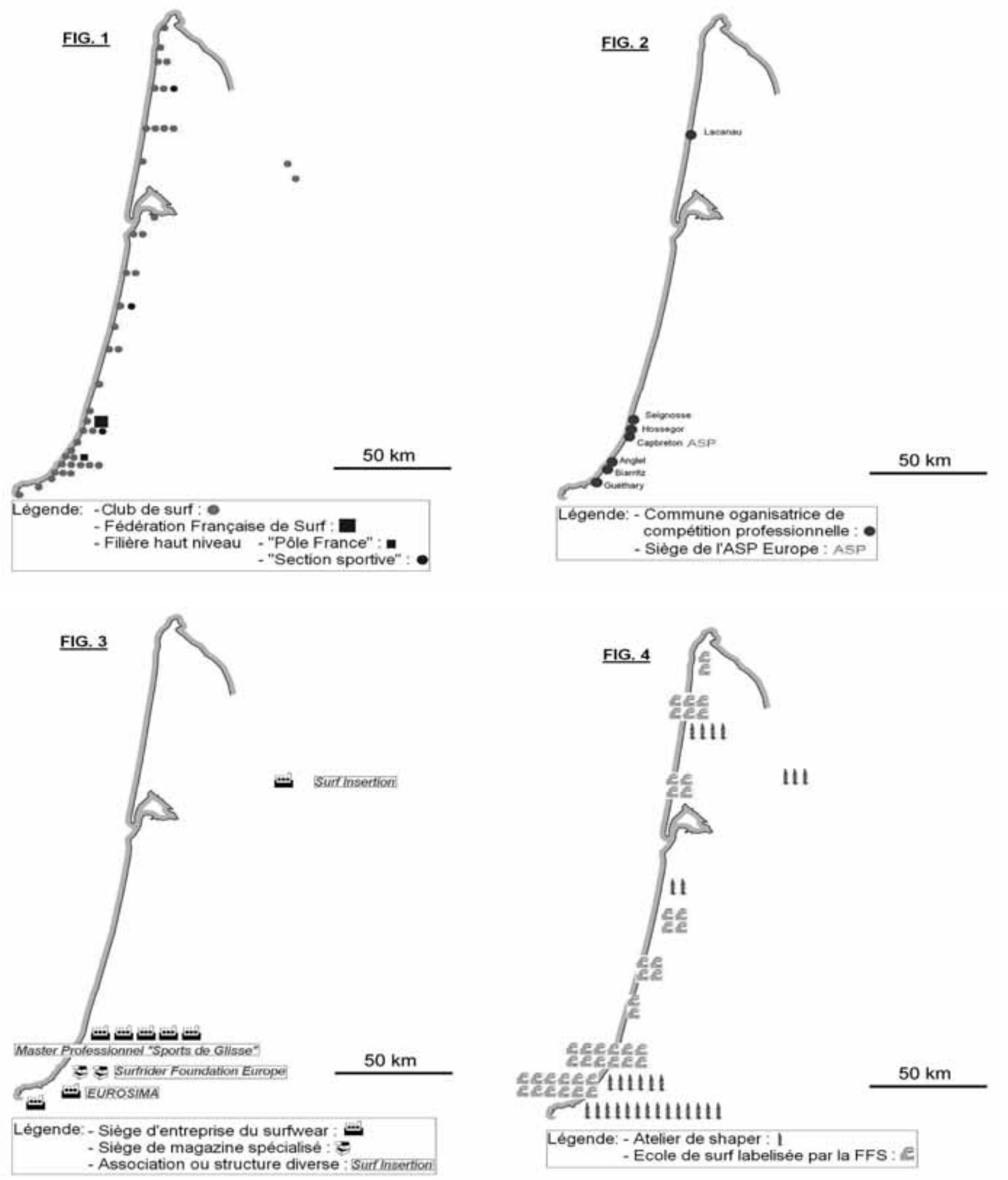

Cartes conçues par l'auteur à partir de données de la FFS (fig. 1 et fig. 4). 
les clubs sont localisés de manière hétérogène au sein des trois départements (fig. 1). Ce n'est en conséquence pas un hasard si les actions municipales à l'égard du surf y sont les plus dynamiques, preuve que l'histoire de la pratique, notamment, et la proximité géographique ravivent des concurrences territoriales fortes.
Une commune littorale ne peut donc maintenir ou acquérir, par l'intermédiaire du dynamisme politique de l'équipe municipale et/ou de son maire, un statut de ville de surf qu'à partir du moment où les élus locaux y voient un intérêt (Bourdieu, 1994 : 149), lequel favorise cette relation d'interdépendance entre un territoire et ses pro-

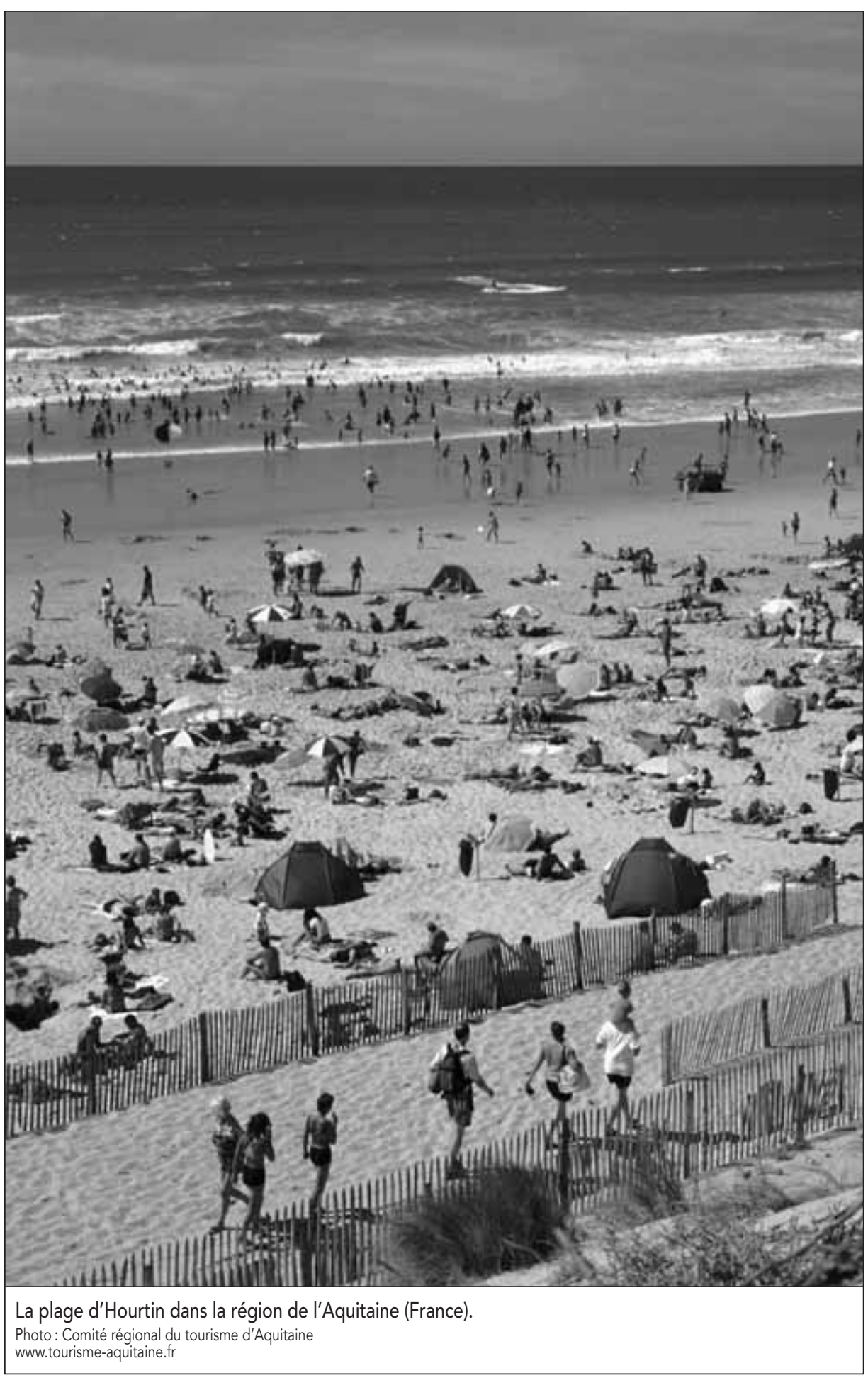

pres singularités, et l'univers du surf. C'est cet intérêt qui est le moteur du dynamisme des élus et des actions politiques ${ }^{6}$. On le voit, les usages politiques possibles pour un élu local en Aquitaine au sujet du surf sont multiples. Cet intérêt passe le plus souvent par la valorisation de l'identification de la commune afin d'accroître l'offre touristique locale. Une telle stratégie pour une équipe municipale s'envisage cependant au regard des atouts disponibles localement et en fonction des actions des autres communes.

\section{Un même support, des productions d'externalité différenciées}

Les pouvoirs locaux justifient des utilisations différenciées de la vague en tant que substrat idéologiquement construit. La Chambre de commerce et d'industrie de Bayonne-Pays basque rappelle d'ailleurs explicitement que le surf est une composante tout à fait majeure dans l'économie locale: "première région européenne de la glisse ", le surf est devenu une activité incontournable à tel point que « la glisse, c'est au Pays Basque " ${ }^{7}$. Jean-Pierre Augustin (1994: 110) dénote, dès 1994, qu'un "processus de 'surfurbia' est en cours autour des agglomérations de la Côte Basque et des stations océanes proches de Bordeaux". Chaque municipalité révèle et valorise cependant les vagues de ses plages et le surf en fonction des conditions de pratique historiquement constituées, ainsi que des ressources naturelles à proprement parler. Un élément explicatif réside notamment dans l'iconographie utilisée par les communes et les offices de tourisme. Une analyse systématique de ces supports permet de préciser le rapport étroit qui existe entre les «immatérialités naturelles » et le sens spécifiquement construit par les acteurs, autrement dit les composantes idéelles. En effet, Guy Di Méo (1999: 150) argue que «le territoire et son corollaire identitaire s'affirment de plus en plus comme des valeurs idéelles ». Le surf sur la côte aquitaine répond ainsi, selon Jean-Pierre Augustin, à certaines exigences :

Forme première des sports de glisse, le surf valorise le rapport à la nature, le contact avec les éléments et la prise de risque. Or l'Aquitaine est un vaste réservoir d'espaces naturels, avec notamment plus de deux cents kilomètres de littoral, et les vagues de l'océan offrent l'exutoi- 
re exceptionnel correspondant aux attentes des pratiquants. Les collectivités locales et les médias ont bien compris le parti quills pouvaient tirer d'une telle situation, en mettant en valeur le surf en tant que pratique, mais aussi comme une image positive pour promouvoir les entreprises ${ }^{8}$.

Mais c'est à partir des conditions locales de l'espace social et des ressources disponibles relatives à l'univers du surf (pratique, emplois directs, pouvoirs internes, événementiel...), puis de l'espace politique (la position d'un maire ou d'un élu qui s'autorise - ou non à croire et agir), autrement dit à partir de préoccupations sociologiques émanant du " contexte ", que sont mises en évidence les stratégies différenciées des élus locaux. Les cas concrets et empiriques qui suivent montrent bien que le surf, et donc son support naturel, la vague, sont utilisés et valorisés différemment à l'échelle communale. Alors que seuls les initiés à la pratique du surf peuvent discerner la qualité des vagues et les catégoriser (à tout le moins subjectivement), une vague reste une vague pour le néophyte. II peut cependant paraître étonnant de voir que certaines communes françaises ont ce statut de ville de surf, sont du moins perçues comme telles, alors que la qualité des vagues y est, pour ainsi dire, "moyenne " sur une "échelle de qualité " nationale. C'est par exemple le cas de Biarritz qui doit son statut de "capitale européenne du surf » à l'historicité de la pratique sur ses plages et à la force des productions d'externalité assurées par la municipalité.

Biarritz est le « berceau historique du surf en Europe». Le surf, "apparu» en 1956, y "est un mode de vie [...] inscrit dans les gènes de cette ville", selon les mots de Michel Veunac, adjoint au maire chargé de la communication. Aussi, ce sport est-il maintenu à un rang élevé dans la politique sportive et de communication de la ville (Guibert, 2003: 48). Si Biarritz est le «royaume du surf » en Europe, à tout le moins affirmé comme tel par les élus biarrots, c'est que ceux-ci peuvent légitimement s'appuyer sur l'historicité de la pratique sur leurs plages. Peu axé sur le pôle compétitif, c'est le Biarritz Surf Festival, largement soutenu par la municipalité - à hauteur de 50000 euros pour l'édition 2005 par exemple -, qui structure avec force l'identification de Biarritz comme ville de surf. Pratiques coutumières originaires d'Hawaii, musique traditionnelle polynésienne, pratique du surf «à la mode » des années 1950, c'est la " culture surf » qui est mise en exergue. Cette imposition symbolique, un peu comme si seule cette culture était digne d'intérêt, écarte du festival toutes les modalités de pratique qui ne correspondent pas aux attentes des organisateurs. C'est « l'authenticité » de la pratique à Biarritz qui est valorisée grâce à l'historicité locale du surf. Réinventé, le surf est ici exploité par des entreprises privées (les multiples sponsors) ainsi que par l'administration municipale, au profit de la visibilité de la commune et de sa reconnaissance par l'extérieur, attestant sur ce point l'orientation plus communicationnelle que sportive de la politique menée. Mais cette volonté des pouvoirs locaux de valoriser ce festival aux dépens de compétitions internationales comme par le passé (Biarritz a été une ville hôte d'une étape du championnat du monde professionnel dans les années 1980 et 1990) est liée aux ressources naturelles disponibles sur les plages biarrotes. C'est parce que les vagues n'y sont pas aussi puissantes et régulières que sur les plages du sud des Landes, notamment, que la vague est utilisée de manière singulière, dans le but de maintenir la station balnéaire au rang des principales villes de surf en France.

Plus au nord, en Gironde, la station balnéaire de Lacanau est depuis plus de vingtcinq ans un lieu privilégié en France pour l'organisation de compétitions nationales et internationales. Le Lacanau Pro, créé en 1979, est la plus ancienne étape du championnat du monde professionnel en Europe. La compétition y est perçue comme le principal, voire l'unique élément structurant à long terme. Les mots du secrétaire général du Lacanau Surf Club sont d'ailleurs tout à fait explicites quant au dynamisme de la municipalité actuelle:

C'est vrai que, quand nous on veut organiser une compétition, la municipalité répond toujours "présent». Que ce soit au niveau des autorisations, des subventions, du prêt de matériel, de banderoles, etc. ils sont toujours là. [...] Le surf fait partie des grosses activités ici à Lacanau et la municipalité le perçoit très bien. D'ailleurs, on leur dit sans cesse que Lacanau est connue grâce au surf, pas à l'équipe de foot! Donc ils le savent bien, ils se rendent compte que le surf est prioritaire, le surf est l'élément moteur ici!
Finalement, le surf à Lacanau ne s'envisage pas comme une pratique de plage ou de simple loisir. La communication de la ville de Lacanau en faveur du surf se définit donc par la modalité dominante de la compétition, dont la vague est le support privilégié. Les retombées médiatiques d'une compétition comme le Lacanau Pro témoignent des enjeux en termes de communication et d'attrait touristique. L'examen des articles dans la presse écrite rend compte des effets médiatiques de la manifestation. Chaque année, le Lacanau Pro est évoqué dans les dépêches des agences de presse, dans la presse régionale, dans la presse nationale quotidienne, dans les magazines sportifs, dans la presse spécialisée, dans la presse nationale et spécialisée étrangère (États-Unis, Australie, Espagne, Grande-Bretagne, Afrique du Sud, etc.) ${ }^{9}$.

Les vagues et le surf sont donc utilisés par les pouvoirs locaux en fonction des finalités définies au préalable, à savoir le type de tourisme, le type de manifestation, le message, la valorisation, etc. qu'il convient de définir. II en résulte des représentations qui jouent sur les catégories mentales de la population locale et des touristes alors que, initialement, le support naturel est sensiblement le même. C'est ainsi que les vagues représentées sur les affiches des éditions du Biarritz Surf Festival sont petites et lentes, illustrant du même coup la volonté de recruter des spectateurs aux caractéristiques sociales singulières. Organiser le surf à travers cette modalité, le recours au «noble passé » ou à «l'éternel hier », permet de positionner Biarritz de manière originale dans l'espace des «villes de surf ", comme étant la seule qui puisse légitimement prétendre à «l'authenticité ». Cette vision du surf sous-entend un recrutement social plus familial et moins «jeune » que celui propre aux manifestations organisées à Lacanau en Gironde ou à Hossegor dans les Landes, lors de compétitions professionnelles.

À l'inverse, les vagues représentées sur les affiches de ces dernières sont grosses et puissantes, procurant un effet symbolique de "danger». Dans ce second cas, la puissance des éléments naturels est mise en exergue, alors qu'à Biarritz la vague est le support de la "culture surf » et du passé prestigieux de 
Encadré 1

\section{À Seignosse, "La vague est là »}

La rhétorique singulière employée ici en couverture de la revue municipale Seignosse Magazine No 1 à l'été 2001 met en évidence, pour la municipalité, le poids de la vague et du surf comme sources de profits potentiels (économiques, touristiques, identification). La vague y est présentée comme ressource exclusive, laissant penser que, si «la vague est là », elle n'est pas ailleurs.
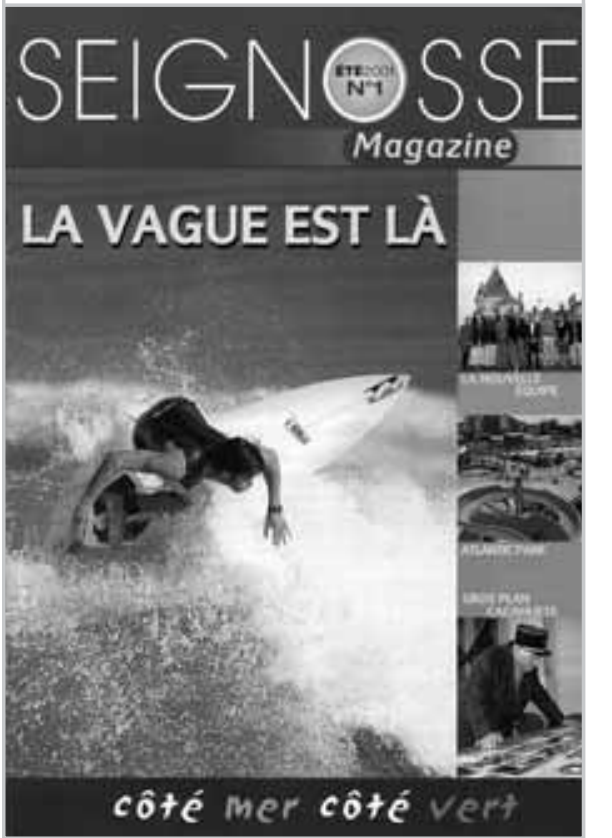

Couverture d'un magazine.

Photo: Seignosse Magazine № 1, été 2001.

la station. Les sens conférés à ces deux types de manifestation s'opposent en de nombreux points et la vague contribue à alimenter cette différence idéelle (lentrapide, soft-hard, petite-puissante, etc.). Dans le premier cas, tel que mentionné, la vague est le support de la "culture surf » qui vise à valoriser le passé prestigieux de Biarritz; dans le second, elle est le support de la performance sportive en compétition. Bien que répondant à des logiques différentes, les festivals et les compétitions professionnelles sont les événements les plus effectifs dans la construction d'une identification de ville de surf.

\section{Recherche de différentiation, politique de communication et retombées touristiques}

Les compétitions professionnelles de surf, très localisées en France (Lacanau, Hossegor, Seignosse, Capbreton, Anglet et Biarritz uniquement), et les festivals sont des ressources territoriales historiquement datées qui sont étroitement imbriquées dans l'action politique municipale. Ces événements deviennent des ressources territoriales communales à partir du moment où se définit un processus d'élaboration, de construction et de valorisation par la municipalité. C'est parce que la ressource territoriale est dépendante d'une valorisation datée dans le temps (l'inscription de l'événement au "programme politique ${ }^{10}$ ) et située géographiquement qu'elle est un moyen privilégié de lutter contre la concurrence. Pour une municipalité, communiquer sur la vague, autrement dit construire et valoriser ce type de ressource, exige de fait une recherche de différentiation qui permet in fine au territoire communal de se démarquer. La vague ravive en quelque sorte la proximité géographique ou, plus trivialement, des «querelles de clocher».

Seignosse, petite commune du sud des Landes, se situe près de Hossegor, commune plus prestigieuse eu égard à un long passé de compétitions professionnelles, où se situe notamment le siège de la FFS. C'est parce que Seignosse ne peut pas faire valoir un passé et une légitimité «surfiques » aussi prestigieux que Hossegor que la stratégie employée par le maire, Ladislas de Hoyos - comte d'origine autrichienne et journaliste de télévision à la retraite -, est de rendre exclusive la qualité de vague de ses plages pour la pratique du surf (voir encadré 1). Cet exemple montre à quel point la vague a un pouvoir de "fragmentation » territoriale, pour reprendre le mot d'Hervé Gumuchian (2000: 101-102), sur les communes du littoral aquitain. Autrement dit, l'espace du surf (la pratique, les compétitions, les festivals) permet difficilement aux communes de définir des politiques cohérentes et consensuelles. Les retombées sont perçues par les municipalités comme des vecteurs de développement touristique et économique qu'il convient de conserver, aux dépens des communes voisines.
Le surf permet, grâce à la portée médiatique que connaissent les compétitions internationales et les festivals, notamment (retransmissions télévisées, articles dans la presse sportive locale et spécialisée, Internet), un développement accéléré du tourisme estival. Le nombre de spectateurs lors de chaque édition du Rip Curl Pro dans le sud du département des Landes, plus de 200000 en cumulé selon les organisateurs et les médias pendant une semaine, fin août, sur les plages du sud des Landes est révélateur. Les propos de JeanPierre Dufau, maire de la commune de Capbreton dans le sud des Landes, illustrent un réel enthousiasme quant à la fenêtre médiatique "sans précédent » que lui offre l'organisation d'une compétition professionnelle comme le Rip Curl Pro ${ }^{11}$. En Gironde, le Lacanau Pro, également en août, redynamise le tourisme sur le littoral. À la suite du naufrage du pétrolier Le Prestige à l'hiver 2002 en Espagne, l'été 2003 reste singulier en termes de communication. Après un mois de juillet «catastrophique » sur le plan de la fréquentation touristique dans le sud-ouest de la France (les courants marins ont «apporté » du pétrole sur les plages françaises), le quotidien régional Sud-Ouest présente le surf sous le titre "La planche de salut " ${ }^{12}$, comme la ressource miracle, "sauvant la saison" à Lacanau. Pour l'organisateur du Biarritz Surf Festival, "Lacanau était un trou à rats: personne ne connaissait Lacanau. Cette ville a été connue grâce au Lacanau Pro et il faut rendre hommage à ceux qui l'on créé ${ }^{13}$. "

La mise en scène médiatique des villes de surf leur assure une visibilité non négligeable, surtout quand c'est la télévision nationale qui propose des émissions dédiées au surf :

Hossegor, petite ville des Landes, mais surtout haut lieu du surf mondial. Ici, tout tourne autour du surf et en cette fin du mois d'août, le temps s'est arrêté: ils étaient tous ce week-end à la plage pour voir les finales du Rip Curl Pro. Si Biarritz est la ville historique du surf, si Lacanau fut la première station balnéaire dans notre pays où fut organisée une épreuve du circuit professionnel, Hossegor s'est fait une renommée grâce à ses vagues. Si Hossegor est la ville du surf, on y pratique beaucoup d'autres sports de glisse, mais ce weekend, tout le monde était hors de l'eau pour suivre les finales du Rip Curl Pro ${ }^{14}$. 
La télévision est assurément le canal médiatique le plus populaire puisque la retransmission d'un événement "surfistique » touchera des millions de téléspectateurs très éloignés culturellement et géographiquement du surf. C'est effectivement la télévision qui a les plus grandes capacités de faire connaître un lieu et a fortiori une pratique sportive. Christophe Arrondeau, directeur de cabinet du maire de Capbreton, est ravi des retombées médiatiques sur sa commune qu'apporte un événement comme le Grommets Surf Trophy, compétition destinée aux meilleurs juniors mondiaux :

On était très content de voir des jeunes à notre compétition des "Grommets Surf Trophy », c'était sympa de voir des minots sur les vagues. En plus de ça, on avait tous les ans à la télévision le "clin d'œil météo » de France 2, qui est le plus écouté et aussi sur Stade 2 : donc c'est jackpot ${ }^{15}$ !

Les sites Internet des communes et des offices de tourisme du littoral aquitain sont également un bon moyen de communiquer sur le surf. La fréquentation des sites par les internautes (plus de 100000 visites depuis l'existence du site de la ville de Seignosse en deux ans par exemple) montre qu'une municipalité ou un office de tourisme ne peut en faire économie. La communauté d'agglomération de Biarritz-AngletBayonne estime que près de $30 \%$ des réservations estivales sont effectuées par Internet. Pour ce qui est des stratégies à l'égard du surf, là encore elles sont différentes. Dans certains cas $^{16}$, le surf est explicitement mis en évidence, contrairement à d'autres sites où il n'est pas plus mis en valeur qu'une autre pratique sportive. Les sites des communes de Guéthary et de Lacanau sont les plus significatifs: ce sont ceux où, dès la page d'accueil, une photographie de surf paraît au premier plan. Le site de la commune des Pyrénées-Atlantiques est à ce titre révélateur d'une volonté affichée de promouvoir le surf. Une photographie de surfeur est d'ailleurs apposée sur celle d'un pratiquant de pelote basque. Le site propose en outre un lien vers «la météo des vagues ».

Que ce soit à l'aide de festivals ou de compétitions professionnelles, valorisés par la presse, la télévision ou Internet, les manifestations de surf sur la côte Aquitaine s'inscrivent dans les politiques municipales. Elles diversifient les retombées médiatiques, elles-mêmes susceptibles d'élargir l'offre et les profits touristiques. Or, l'histoire de la pratique compétitive en France montre que les conditions climatiques optimales ne sont pas toujours présentes lors des manifestations.

\section{Encadré 2}

\section{La Maison de la Glisse à Lacanau : une « vitrine du surf » luxueuse}

Idéalement située sur la dune, face à l'océan et aux principaux spots de Lacanau, la « Maison de la Glisse » est un immeuble de 450 mètres carrés visible du remblai et des plages. L'aménagement intérieur est représentatif des moyens financiers investis par les collectivités territoriales : bureaux, accueil, coins «salon », jeux et, au sous-sol, une «salle de gym » digne d'un établissement spécialisé de « remise en forme » avec de nombreux équipements de musculation, des vestiaires et des locaux pour entreposer les planches et les combinaisons de surf.

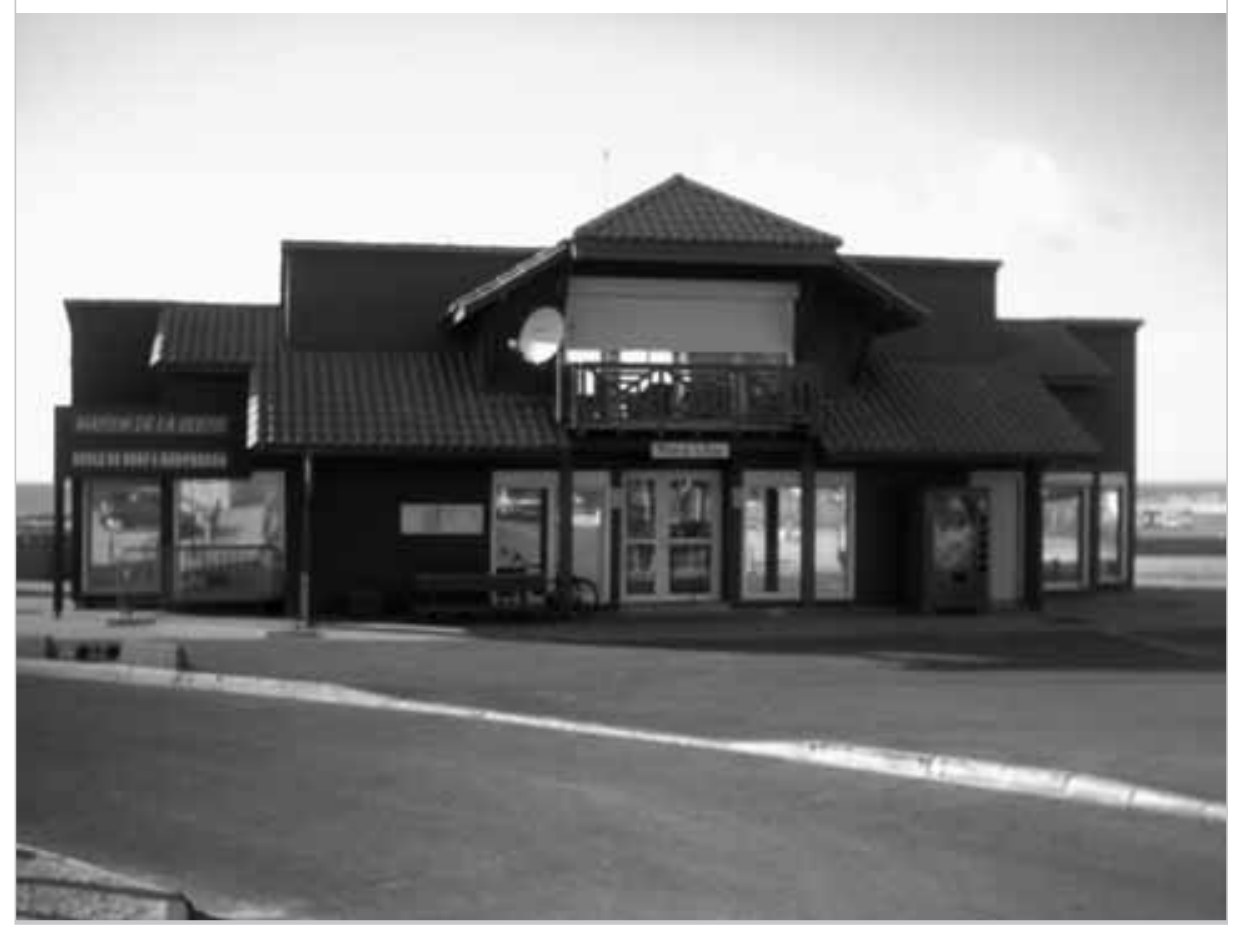

Photo : Christophe Guibert

\section{Faire durer l'éphémère}

Compétitions professionnelles annulées en raison du manque de vagues ou manifestations reportées afin de proposer des conditions de pratique meilleures, le surf reste dépendant de circonstances qui ne peuvent être assurées par les municipalités. Aussi, le volontarisme politique doitil être d'autant plus grand que l'éphémère augmente, d'où la condition nécessaire d'orienter les projets politiques de manière continue. Chaque année, les collectivités locales se doivent de lutter pour "récupérer » une compétition susceptible d'alimenter l'image et la notoriété précédemment acquises. Autrement dit, le processus de valorisation d'une ressource territoriale liée au surf n'est jamais dé- fini sur le long terme. Toutefois, deux exceptions localisées remettent en question la caractéristique du surf, tenant au fait que la pratique de ce dernier ne nécessite pas d'infrastructure. Afin de faire durer dans le temps une activité trop limitée à une pratique saisonnière et dépendante des conditions climatiques, la municipalité de Lacanau en Gironde, avec l'appui du Conseil général de Gironde et du syndicat mixte local, a souhaité créer une "Maison de la Glisse » dont l'ouverture a été effective en 1990. Seul établissement destiné à héberger une structure associative de surf de cette envergure en France, la Maison de la Glisse constitue, avec le Lacanau Pro, la « vitrine » du surf de la ville (voir encadré 2). 
La municipalité de Biarritz s'inscrit dans une logique similaire d'identification territoriale durable. Elle a examiné lors d'un conseil municipal en septembre 2004 le projet de réalisation de la "Cité du Surf ». Cette idée, qui existe déjà depuis plusieurs années, prend de la consistance avec la décision récente de lancer une procédure de concours pour la désignation de l'équipe de maîtrise d'œuvre. Ce projet illustre la volonté de la municipalité de continuer à légitimer auprès des surfeurs, des habitants et des touristes, une image structurante de ville de surf incontournable en France et en Europe. Joël de Rosnay, un des premiers surfeurs français, également chercheur à la Cité des Sciences de la Villette à Paris et auteur de nombreux ouvrages de vulgarisation scientifique, met au profit de la municipalité son aura médiatique dans l'univers du surf. II est ainsi désigné par la municipalité "animateur du Comité scientifique d'orientation de la Cité du Surf ». L'établissement, implanté sur une superficie de plus 3700 mètres carrés, sera un élément puissant de différenciation territoriale. L'achèvement des travaux est prévu pour la fin de l'année 2007. II s'agira d'un véritable musée dédié au surf et à la mer - 700 mètres carrés seront consacrés uniquement à " l'histoire du surf depuis ses origines ${ }^{17}$-; seule la commune de Biarritz possède une légitimité historique suffisante en France pour proposer un tel équipement. Le coût de l'opération, estimé à 8,38 millions d'euros et inscrit dans la convention spécifique du Pays basque ${ }^{18}$, témoigne de l'envergure du projet qui comprendra :

quatre espaces principaux : un espace accueil ; un centre de ressources composé d'une salle d'exposition permanente sur le surf, la glisse et l'océan, d'une galerie info-expos, d'un atelier pour enfants complété par une aire de pique-nique; une aire de détente et de rencontres avec café-restaurant thématique, terrasse et kiosque de surfeur ; un espace ludique avec images en 3D et jeux virtuels. L'objectif étant d'inviter les jeunes à comprendre l'océan, notamment pour mieux le protéger ${ }^{19}$.

Le surf ainsi exploité devient un outil politique et touristique visant à marquer un territoire afin que ce dernier maintienne ou développe un statut de ville de surf ou de « lieu rayonnant »20 (Sibertin-Blanc, 2003 : 103).

\section{Conclusion}

La vague est un élément qui permet de différencier et de catégoriser les actions des pouvoirs locaux sur la côte Aquitaine. Les singularités de cette composante naturelle, tout comme celles de l'univers du surf, rendent les actions politiques très variables. Les mécanismes de valorisation de cette ressource éphémère se définissent localement en fonction du contexte et des intérêts perçus par les élus locaux. C'est la malléabilité du surf ainsi que la plasticité du support de cette pratique, la vague, qui facilitent finalement des usages politiques spécifiques. Aussi, les choix politiques qui visent à définir des identifications telles que celles qui sont analysées dans ce travail constituent-ils un rempart à la normalisation des politiques municipales. Quoiqu'il en soit, il s'agit en dernier lieu pour les pouvoirs locaux de contrôler et de pérenniser l'identification territoriale de la ville dans le champ des "villes de surf ", grâce à l'instrumentalisation de la vague, avec la finalité non avouée d'être élue la plus visible et la plus reconnue. Le processus de valorisation d'une ressource territoriale par les pouvoirs locaux soulève donc la question de l'intérêt pour ces mêmes élus dans le champ politique.

Cet examen permet de lutter contre l'illusion d'unification des politiques des collectivités locales par le simple label réitéré mécaniquement de "politique sportive " qui n'a bien souvent de sportive que le nom. Manifestement, I'identification territoriale d'une commune grâce à une activité sportive passe plus par la définition d'une politique centrifuge, visant à accroître une visibilité tournée vers l'extérieur, que par la définition d'une politique centripète en faveur des adeptes locaux. Toutefois, définir et maintenir une politique sportive peut servir de préalable à une politique de communication ou à une politique touristique. Mais c'est parce que l'événementiel trouve de forts impacts dans le champ médiatique qu'il est considéré dans le champ politique. On voit bien que des municipalités du littoral aquitain, comme c'est le cas à Biarritz ou à Lacanau, justifient leur politique municipale de développement du processus d'identification territoriale grâce aux ressources antérieurement présentes sur leur commune respective. Ces dernières doivent être suffisamment influentes aux yeux des élus sur le plan médiatique. Ces dyna- miques politiques à l'échelle locale, qui s'inscrivent dans une logique de marquage du territoire à des fins de légitimité touristique, ne prennent que rarement en compte les besoins des pratiquants dont les exigences sont, aux yeux des élus, moins porteuses politiquement et médiatiquement. Les représentations des équipes municipales à l'égard des surfeurs s'apparentent encore trop, en France, à ce qu'Alain Loret appelle la "contre-culture » sportive (Loret, 1996). Les propos de responsables de club, respectivement celui de Lacanau et celui de Biarritz, communes où les municipalités font durer l'éphémère grâce à des réalisations immobilières (la Maison de la Glisse et la Cité du Surf), illustrent le décalage entre la communication par le surf et une politique sportive municipale:

Ils [la municipalité] ont décidé de faire la Maison de la Glisse. Ils ont chargé un architecte de faire ça, sans notre avis. Ils n'ont pas pris en compte nos besoins. Nous, on avait l'impression que c'était beaucoup trop tôt, bien trop luxueux! Mais bon, le maire, pour lui, il fallait que ça se voit. Plus qu'un lieu pour les surfeurs, c'est une vitrine, c'est presque du «tape à l'œil »21.

Les surfeurs, ils n'en ont rien à faire. Ils s'en foutent de la Cité. Pour eux, ce sont les vagues qui comptent. Bon, on ira voir, mais c'est tout quoi! Alors après, cette Cité, elle renforce l'image de Biarritz, du surf à Biarritz. Mais plus on parle du surf à Biarritz, plus ça attire du monde et plus il y a de surfeurs sur les spots! Alors les locaux ${ }^{22}$, ils n'aiment pas trop quoi ${ }^{23}$ !

En dernier lieu, on comprend bien que le surf professionnel, les festivals et les infrastructures - comme la Cité du Surf sont sources de profits (économique, touristique, médiatique...) pour la commune, validant sur ce point le statut d'outil politique pour les municipalités dans la lutte symbolique et médiatique entre les communes. Devenir le maire d'une commune "dynamique ", « jeune ", "qui bouge ", " dont on parle » apporte assurément des profits sociaux et symboliques, réinvestissables à bon compte dans le champ politique local, voire à une échelle politique plus élevée.

Christophe Guibert est docteur en sociologie, Centre Nantais de Sociologie. 


\section{Notes}

1 Les effets de symbolisation du territoire par le sport sont nombreux et les exemples significatifs illustrent l'influence et l'efficacité du sport en termes "d'image»: le football à Marseille ou à Madrid, l'automobile avec les «24 Heures du Mans » ou les « 500 Milles d'Indianapolis ", l'équitation à Saumur, le basket-ball féminin à Valenciennes, la voile aux Sables d'Olonne avec le Vendée Globe, etc.

2 Cet extrait, issu d'un entretien effectué en 2001 dans les locaux du magazine Surf Session, corrobore nombre d'articles parus dans la revue.

3 Le Monde "Trois questions à Jean-Pierre Augustin », 13 décembre 2000.

4 Depuis l'instauration en 1984 de la formation au brevet d'État de surf, délivré par la Fédération française de surf, près de 500 surfeurs sont devenus moniteurs. Seuls 200, selon les statistiques fédérales, enseignent chaque année dans les clubs et les écoles privés, en grande majorité lors de la période estivale (Guibert, 2002 : 116).

5 L'espace du surf s'envisage théoriquement à l'instar de l'espace des sports évoqué par Pierre Bourdieu (1987 : 203-216).

6 Ce qui revient finalement à dire, avec Max Weber, que "l'entreprise politique est nécessairement une entreprise d'intérêt » (Weber, 2001 : 164).

7 Le développement socioéconomique engendré par le surf est convoqué de manière exhaustive sur la page "Glisse » du site Internet de la Chambre de commerce et d'industrie de Bayonne-Pays basque. (Source: [www.bayonne.cci.fr].)

8 Le Monde "Trois questions à Jean-Pierre Augustin », 13 décembre 2000.

9 Une liste exhaustive des retombées du Lacanau Pro dans la presse écrite a été réalisée à l'issue de l'édition de 1992 par les organisateurs. (Source: Dossier de presse du Lacanau Pro, 1992.)

10 On entend par mise au programme politique le processus lié à l'intervention du politique au sujet d'un problème ou d'un événement digne d'intérêt et perçu comme tel par un élu (Padioleau, 1982: 25).

11 «Les cinq communes constituées en syndicat intercommunal ont apporté leur soutien actif aux organisateurs du Rip Curl Pro dès la première manifestation en 1987. Chaque année, le SIVOM [Syndicat intercommunal à vocations multiples] Côte-Sud fait bénéficier cette manifestation de la plus forte subvention qu'il accorde aux événements et manifestations de tous genres sur les cinq communes. Cela permet de mesurer la place que tient le Rip Curl Pro dans l'esprit de nos élus! Ils sont en effet conscients que c'est l'événement le plus fréquenté chaque année, qui génère des retombées médiatiques sans précédent. Cette épreuve sportive incarne également très bien l'image de nos stations associant la recherche d'une certaine qualité de vie, certains diraient dépassement de soi, au contact de l'océan et le respect de l'environnement, la convivialité, la dimension humaine, en un mot : notre art de vivre. " Document de présentation du programme officiel du Rip Curl Pro, 1996.

12 Sud-Ouest, 11 août 2003.

13 Extrait d'un entretien effectué en avril 2002.

14 «Minuit Sport », TF1, août 1992.

15 Entretien effectué en avril 2001 à la mairie de Capbreton.

16 Cette analyse a été effectuée au premier semestre de l'année 2001.

17 Sud-Ouest, "Prélude à la cité du surf », 8 avril 2005.

18 Convention marquant un partenariat entre l'État, la région, le département et le Conseil des élus du Pays basque, signée en décembre 2002. Elle prend en compte des dépenses (400 millions d'euros) liées à l'environnement, à l'urbanisme, au tourisme, à la formation et aux industries et à la défense de la langue basque.

19 Biarritz Magazine, "La Cité du Surf émerge », n¹35, novembre 2004, p. 10.

20 "Le lieu rayonnant », rare par définition, est évoqué relativement à l'instrumentalisation de la culture à des fins de marketing territorial par Mariette Sibertin-Blanc (2003 : 103).

21 Entretien effectué en avril 2004 auprès d'un ancien dirigeant du Lacanau Surf Club.

22 «Local only », autrement dit, locaux seulement, est affiché devant le parking qui surplombe les locaux du principal club de Biarritz, près de la plage de la Milady.

23 Entretien effectué dans les locaux du Biarritz Association Surf Club, avril 2005

\section{Bibliographie}

Augustin, Jean-Pierre (1994), « Le littoral aquitain, de la station balnéaire à la station surf », Surf Atlantique, les territoires de l'éphémère, Maison des Sciences de l'Homme d'Aquitaine (MSHA), Bordeaux, p. 97-112.

Augustin, Jean-Pierre (1997), «L'émergence des stations surf sur le littoral aquitain ", Les Cahiers Espaces, n 52, "Tourisme et sport », p. 120-127.

Bourdieu, Pierre (1987), Programme pour une sociologie du sport, Choses Dites, Paris, Minuit, p. 203-216.

Bourdieu, Pierre (1994), Un acte désintéressé est-il possible?, Raisons pratiques, Paris, Seuil, p. 147-171.

Bourdieu, Pierre (2000), Propos sur le champ politique, Presses universitaires de Lyon.

Callède, Jean-Paul (2002), "Quelques logiques de développement sportif en Aquitaine", dans Dominique Charrier et Christophe Durand, Le financement du sport par les collectivités locales. Des ambitions politiques aux choix budgétaires, tome 2, Voiron, Presses universitaires du sport, p. 206-211.

Corneloup, Jean, Malek Bouhaouala, Cécile Vachée, et Bastien Soulé (2000), «Formes de développement et positionnement touristique des espaces sportifs de nature », Loisir et société, vol. 24, n¹, p. 21-46.

Di Méo, Guy (1999), «Introduction », Séminaire Le lien social «Territoires », Maison des Sciences de l'Homme Ange-Guépin, Nantes, p. 141-152.

Elias, Norbert (1998), Sport et civilisation, Paris, Fayard.

Gardinier, Alain (2004), Les Tontons Surfeurs, aux sources du surf français, Biarritz, Atlantica.

Guibert, Christophe (2002), « Le surf », dans RUNOPES (Réseau universitaire d'observation et de prospective de l'emploi sportif), L'emploi sportif en France: Situation et tendances d'évolution, AFRAPS-RUNOPES (Association francophone pour la recherche en activités physiques et sportives), p. 115-117.

Guibert, Christophe, (2003), «De l'authenticité de la pratique du surf à Biarritz ", Espaces, Tourisme, Loisirs, n²09, p. 48-52.

Guibert, Christophe (2004), «De la nécessité de l'analyse à l'échelle du 'local'. Quelques cas de politiques municipales ", dans Patrick Bouchet et Claude Sobry, Management et marketing du sport: Du local au global, Villeneuve d'Ascq, Septentrion, p. 217-234.

Guibert, Christophe (2005), Identifications territoriales et usages politiques du surf sur la côte aquitaine. Des villes de surf sans surfeurs?, Doctorat de sociologie, sous la direction de C. Suaud, Université de Nantes, 453 p.

Gumuchian, Hervé (2000), «Le processus de fragmentation socio-territoriale en cours. Quelles implications ", Actes du colloque "Tourisme sportif et territoires: Développement et gestion des espaces de pratiques sportives de pleine nature ", Montagnes méditerranéennes, $\mathrm{n}^{\circ} 11$, p. 101-102.

Gumuchian, Hervé et Claude Marois (2000), Initiation à la recherche en géographie. Aménagement, développement territorial, environnement, Paris, Anthropos.

Gumuchian, Hervé et Bermard Pecqueur, (2004), "La notion de ressource territoriale », Montagnes méditerranéennes, $n^{\circ} 20$, p. 4.

Le Bart, Christian (1999), « Les politiques d'image: Entre marketing territorial et identité locale », dans Richard Balme, Alain Faure et Albert Mabileau, Les nouvelles politiques locales, Paris, Presses de Sciences Po, p. 415-429.

Loret, Alain (1996), Génération glisse. Dans l'eau, l'air la neige... La révolution du sport des «années fun », Paris, Autrement.

Padioleau, Jean-Gustave (1982), L'État au concret, Paris, Presses universitaires de France.

Pecqueur, Bernard (2000), Le développement local, Paris, Syros.

Sibertin-Blanc, Mariette (2003), «L'inscription d'une politique publique sur les territoires métropolitains : Les lieux culturels et la structuration des agglomérations ", dans Maria Gravari-Barbas et Philippe Violier (dir.), Lieux de culture, culture des lieux, Presses universitaires de Rennes, p. 103-112.

Weber, Max (2001), Le savant et le politique, Paris, Plon. 